\title{
Performance of a reentrant cavity beam position monitor
}

\author{
Claire Simon, Michel Luong, Stéphane Chel, Olivier Napoly, Jorge Novo, and Dominique Roudier \\ DSM/Irfu/SACM, CEA/Saclay, F-91191 Gif-sur-Yvette, France \\ Nelly Rouvière \\ CNRS, IN2P3-IPN/Orsay, F-91406 Orsay, France
}

Nicoleta Baboi, Nils Mildner, and Dirk Nölle

DESY, D-22603 Hamburg, Germany

(Received 28 March 2008; published 26 August 2008)

\begin{abstract}
The beam-based alignment and feedback systems, essential operations for the future colliders, require high resolution beam position monitors (BPMs). In the framework of the European CARE/SRF program, a reentrant cavity BPM with its associated electronics was developed by the CEA/DSM/Irfu in collaboration with DESY. The design, the fabrication, and the beam test of this monitor are detailed within this paper. This BPM is designed to be inserted in a cryomodule, work at cryogenic temperature in a clean environment. It has achieved a resolution better than $10 \mu \mathrm{m}$ and has the possibility to perform bunch to bunch measurements for the x-ray free electron laser (X-FEL) and the International Linear Collider (ILC). Its other features are a small size of the rf cavity, a large aperture $(78 \mathrm{~mm})$, and an excellent linearity. A first prototype of a reentrant cavity BPM was installed in the free electron laser in Hamburg (FLASH), at Deutsches Elektronen-Synchrotron (DESY) and demonstrated its operation at cryogenic temperature inside a cryomodule. The second, installed, also, in the FLASH linac to be tested with beam, measured a resolution of approximately $4 \mu \mathrm{m}$ over a dynamic range $\pm 5 \mathrm{~mm}$ in single bunch.

DOI: 10.1103/PhysRevSTAB.11.082802

PACS numbers: 29.90.+r, 29.27.Eg, 41.85.Qg
\end{abstract}

\section{INTRODUCTION}

For the future superconducting accelerators, a beam position monitor (BPM) installed in a cryomodule with a resolution better than $10 \mu \mathrm{m}$ will be helpful to setup, adjust the machine, and obtain a precise beam handling. Superconducting technology is used for accelerators as the free electron laser in Hamburg (FLASH) [1,2], the $\mathrm{x}$-ray free electron laser (X-FEL) [3] which will be built in Hamburg, and the International Linear Collider (ILC) [4] project. The FLASH linac at DESY is also used as a test facility for the X-FEL and the ILC study under the name TESLA Test Facility-Phase 2 (TTF2). These projects all share the basic design of the accelerating cavity, based on the TESLA technology [5].

The reentrant cavity BPM is specially designed to be connected to superconducting cavities which are particularly sensitive to dust particle contamination, and care must be taken to avoid introducing any source of such contamination. This monitor is composed of a radio-frequency reentrant cavity with a beam pipe diameter of $78 \mathrm{~mm}$, four feedthroughs, and electronics which perform signal processing. Its response is linear and accurate; further it can also measure the beam charge.

A first prototype of a reentrant BPM has delivered measurements at a temperature of $2 \mathrm{~K}$ inside a cryomodule in the FLASH linac. The performance of this BPM was analyzed and the limitations of this existing system clearly identified. The second, installed in a warm section of the FLASH accelerator to be tested with beam, sought to address issues raised during the testing of the first BPM. It was designed to achieve a high resolution down to $10 \mu \mathrm{m}$ and to allow bunch to bunch measurements for the X-FEL or ILC. This second prototype has been qualified with beam, achieving $4 \mu \mathrm{m}$ resolution over a dynamic range of $\pm 5 \mathrm{~mm}$ and the possibility to do some bunch to bunch measurements. The reentrant cavity BPM with its mechanical and signal processing designs, theoretical performance, and the measurements carried out with beam are described in this paper.

\section{REENTRANT CAVITY BPM THEORY}

Coaxial reentrant cavity [6] has been chosen for the beam orbit measurement because of its mechanical simplicity and excellent resolution. This cavity is composed of a mechanical structure which consists of three distinct regions as shown in Fig. 1: beam tube (I), gap (II), and coaxial cylinder (III). It has a small size and a cylindrical symmetry which allows a high precision of the machining. Arranged around the beam pipe, the cavity forms a coaxial line which is short-circuited at the downstream end. The cavity is fabricated with stainless steel as compact as possible. The fixing of the antenna tips to the inner diameter of the cavity over coupled the cavity. Large shunt impedance for the monopole and dipole modes can be obtained with an appropriate shaping of the gap.

Passing through the cavity, the beam excites some electromagnetic fields (resonant modes), which are coupled by four feedthroughs to the outside: two of them determine the 


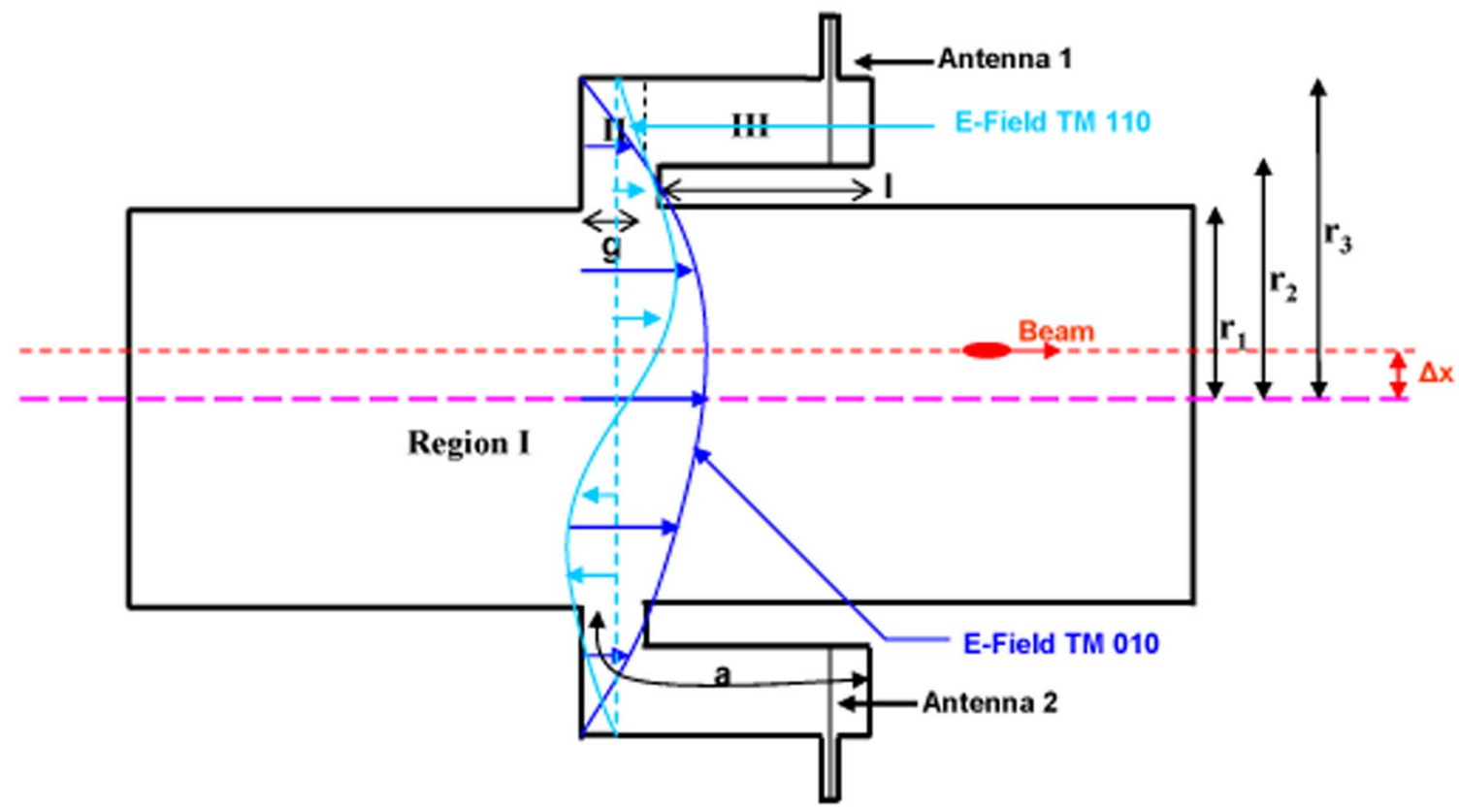

FIG. 1. (Color) Geometry of the reentrant cavity BPM.

horizontal position ( $X$ position) and two others the vertical position ( $Y$ position). The main radio-frequency modes excited by the beam are monopole and dipole modes. The higher order modes are damped much more strongly so their contribution is negligible and the linearity of the measurement is ensured. The monopole mode wavelength $\lambda_{1}$ depends on the length $a_{1}$ of the coaxial cavity:

$$
\lambda_{1} / 4=a_{1}
$$

and the wavelength of the dipole mode is given by

$$
\lambda_{2}=2 \pi\left(r_{m}\right) / \sqrt{1+\left(\pi r_{m} / 2 a_{2}\right)^{2}}
$$

with $r_{m} \equiv\left(r_{3}+r_{2}\right) / 2$.

The exchange of energy between the beam and the cavity depends on the properties of bunch and the geometry of the cavity. The $R / Q$ shunt impedance is given by the following formulas:

$$
R / Q=\frac{V^{2}}{\omega W}
$$

where $\omega$ is the frequency, $V=\left|\int E(z) e^{j k z} d z\right|, k=\omega / c$, and $W$ is the stored energy in the mode, $W=\frac{\varepsilon_{0}}{2}$ 政 $E E^{*} d \tau$.

Each resonant mode has its own shunt impedance. For the monopole mode, the $R / Q$ ratio is a constant contrary to the dipole mode which depends on the beam offset. In the following equations, the $R / Q$ circuit is used.

In single bunch mode [7], the exited energy in the cavity by the beam is calculated by

$$
W \equiv \frac{\omega}{2}(R / Q) q^{2},
$$

where $q$ is the bunch charge.
The power coming from the cavity can be determined with the energy and the rf characteristics for each resonant mode:

$$
P_{\text {out }}=\frac{\omega}{Q_{\mathrm{ext}}} W
$$

The loaded quality factor is determined by the following relation:

$$
\frac{1}{Q_{l}}=\frac{1}{Q_{0}}+\frac{1}{Q_{\mathrm{ext}}}
$$

where $Q_{0}$ is the unloaded quality factor.

In using Eqs. (3)-(5), the output voltage with an impedance $Z$ may be written as

$$
V_{\text {out }} \equiv \sqrt{\frac{\omega_{i}^{2}(R / Q) q^{2} Z}{Q_{\text {ext }}}} .
$$

As shown in Eq. (7), the signal amplitude is proportional to the beam charge, the frequency, the $Q_{\text {ext }}$, and the shunt impedance. The dipole mode (type TM110) voltage is proportional to the intensity and to the distance of the beam from the center axis of the monitor contrary to the signal voltage of the monopole mode (type TM010) which does not depend on the beam position but is proportional to beam intensity. This monopole signal voltage is used for the normalization.

As shown in Fig. 2, the beam position $(X$ and $Y$ ) can be measured by the output voltage from two opposite antennas. The voltage differences $V 1-V 3$ and $V 2-V 4$ correspond to the voltage of the dipole field in the $X$ and $Y$ axis and the sums $V 1+V 3$ and $V 2+V 4$ correspond to the 


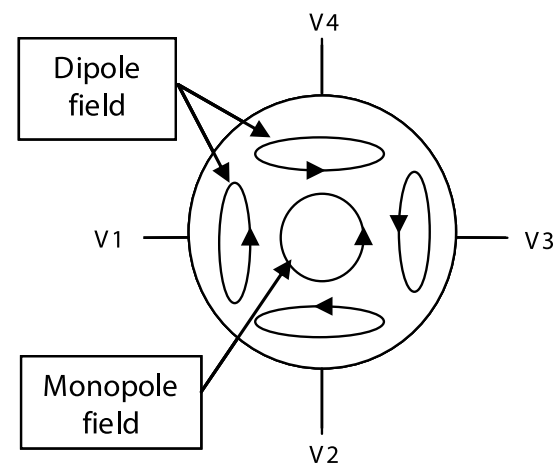

$$
\begin{aligned}
& \mathrm{V} 1=\mathrm{V}(\text { monopole })+\mathrm{V}(\text { dipole }) \\
& \mathrm{V} 2=\mathrm{V}(\text { monopole })+\mathrm{V}(\text { dipole }) \\
& \mathrm{V} 3=\mathrm{V}(\text { monopole })-\mathrm{V}(\text { dipole }) \\
& \mathrm{V} 4=\mathrm{V}(\text { monopole })-\mathrm{V} \text { (dipole }) \\
& \mathrm{X}=\mathrm{L}(\mathrm{V} 1-\mathrm{V} 3) /(\mathrm{V} 1+\mathrm{V} 3) \\
& \mathrm{Y}=\mathrm{L}(\mathrm{V} 2-\mathrm{V} 4) /(\mathrm{V} 2+\mathrm{V} 4) \\
& \mathrm{L}=\text { constant }
\end{aligned}
$$

FIG. 2. (Color) Link between oscillation modes and beam position.

voltage of the monopole field. The $L$ factor is the dipole constant of the beam monitor.

\section{BPM INSTALLED IN A CRYOMODULE}

\section{A. Cavity BPM}

A reentrant cavity BPM, designed for the TTF (Tesla Test Facility) injector [8], was installed in 2004 inside a cryomodule, in an environment where dust particle contamination has to be avoided as shown in Fig. 3.

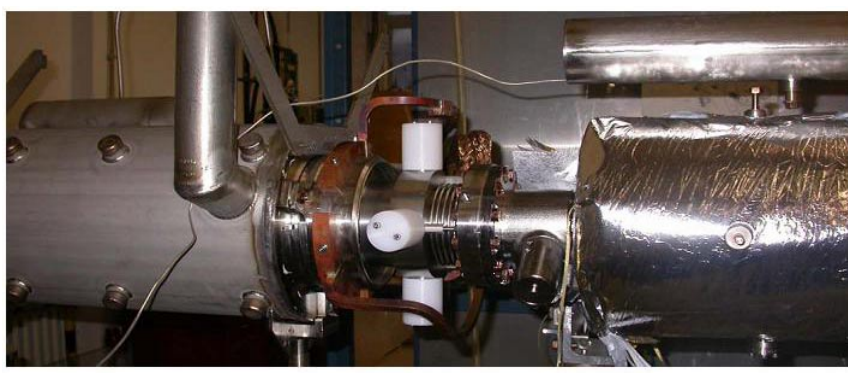

FIG. 3. (Color) BPM installed in a cryomodule with four feedthroughs protected by plastic cylinders during mounting.
The cavity, illustrated in Fig. 4, has been designed thanks to Eqs. (1) and (2) [9]. Its aperture is $78 \mathrm{~mm}$ (aperture of a TESLA cavity), the gap $(g)$ is $8 \mathrm{~mm}$, and the length of the coaxial cylinder is $50 \mathrm{~mm}$.

The rf characteristics (frequencies, $Q_{l}$ and shunt impedance) were calculated with the HFSS code provided by Ansoft Corporation [10]. The first monopole and dipole modes have, respectively, a frequency $f=1.58 \mathrm{GHz}$ and $f=2.01 \mathrm{GHz}$ with a loaded quality factor $Q_{l}=2$ and $Q_{l}=4$ [11]. Because of a very high coupling, the signals are spread out in spectrum. The distinguishing of monopole and dipole modes is not easy and the monopole signal is not, efficiently, rejected to determine the beam position.

\section{B. Signal processing}

The beam position can be measured from the output voltage of a pair of feedthroughs mounted on the opposite sides of the cavity on both axes. Signals detected by the signal processing electronics extract the beam position (displacement) and deliver this information to the acquisition board. Because of the low external quality factor, the single bunch response of the cavity has to be broadened before its acquisition.

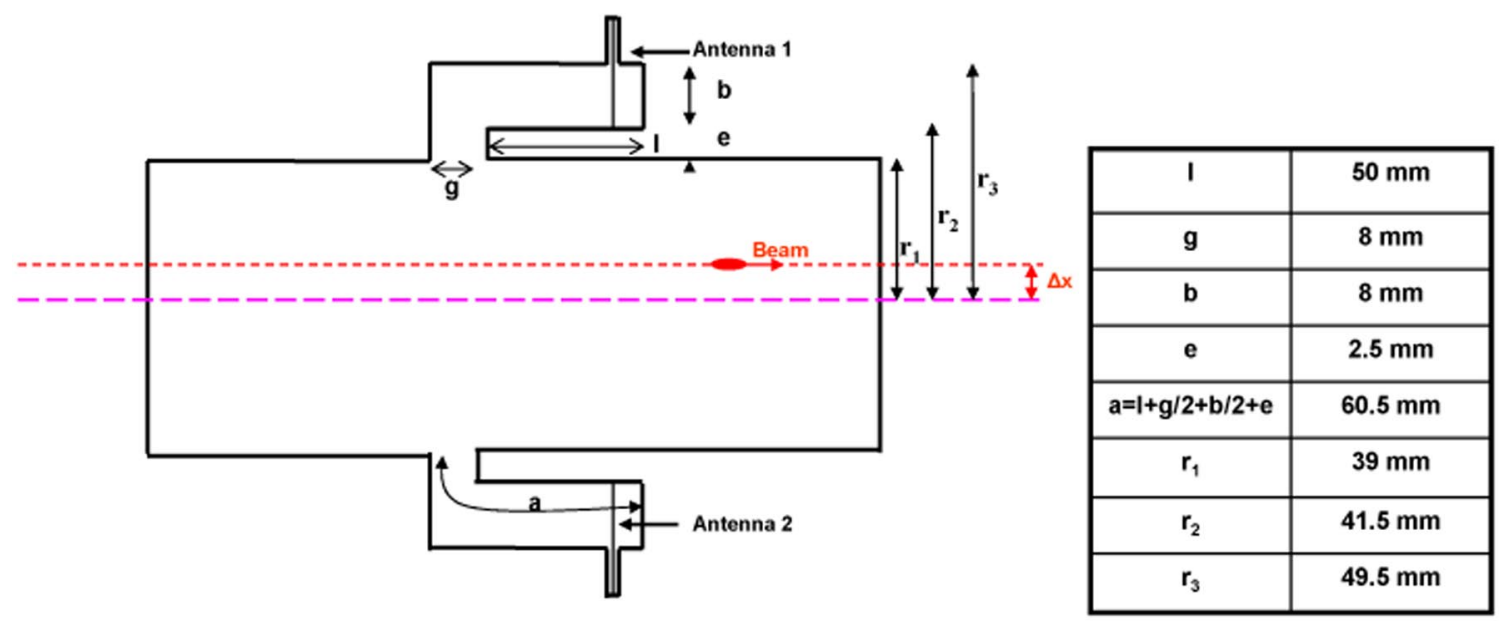

FIG. 4. (Color) Sketch of the reentrant BPM. 
The signal processing uses a single stage downconversion to obtain $\Delta / \Sigma$. The chosen measurement frequency is $650 \mathrm{MHz}$ and is defined by a Bessel bandpass filter used in the signal processing electronics [9]. The $8 \mathrm{MHz}$ bandwidth of this filter defines the bandwidth of the system and determines the time resolution. A $180^{\circ}$ hybrid junction, connected to each pair of opposite antennas with $33 \mathrm{~m}$ of semirigid cables, yields the sum and the difference of rf voltages proportional to the beam current and position. These rf signals are then filtered, amplified, and demodulated with a synchronous detection.

\section{Beam tests on the BPM installed in the cryomodule}

This BPM installed in a FLASH cryomodule was operated in single bunch for a $1 \mathrm{nC} /$ bunch beam. After beam jitter cancellation, the raw rms resolution of the system is estimated around $20 \mu \mathrm{m}$ on the $X$ channel and around $30 \mu \mathrm{m}$ on the $Y$ channel with a linear range about $\pm 1.5 \mathrm{~mm}$ [11]. Moreover, due to the bandpass filter bandwidth, which is only $8 \mathrm{MHz}$, the bunch to bunch measurement on the FLASH linac and X-FEL is impossible with this BPM system [11]. A new design is therefore necessary to achieve a position resolution better than $10 \mu \mathrm{m}$ and have the possibility to perform bunch to bunch measurements.

\section{NEW DESIGN OF A REENTRANT BPM}

\section{A. Cavity BPM}

To improve the time resolution and position resolution of the reentrant BPM which has already proved its capability to be operated inside the cryomodule, a new BPM system was designed.

The principle of the reentrant cavity and the main dimensions are kept: the gap $8 \mathrm{~mm}$, the coaxial cylinder, $170 \mathrm{~mm}$ in length to satisfy the constraints imposed by the cryomodule, $78 \mathrm{~mm}$ for the beam pipe, and $152 \mathrm{~mm}$ in diameter as shown in Fig. 5.

The resolution better than $10 \mu \mathrm{m}$ but also the mechanical feasibility of the structure determined the loaded quality factors, $Q_{l}$, of the monopole and dipole modes. They are not able either to be too high to keep a time resolution around $10 \mathrm{~ns}$ or too low to have a resolution better than $10 \mu \mathrm{m}$. In order to get a higher $Q_{l}$ and therefore a longer signal in time without the need of a Bessel filter for signal shaping, the feedthroughs are moved $31.5 \mathrm{~mm}$ in the reentrant part as illustrated Fig. 5. Some copper-beryllium radio-frequency contacts were welded in the inner cylinder of the cavity to ensure electrical conduction between the feedthrough inner conductor and the cavity, providing a magnetic coupling loop. In the past, a critical point concerned the feedthrough fragility; $50 \%$ of the feedthroughs had to be rejected. With this new design, the machining of feedthroughs is simpler and the final product more robust. Several cryogenic and vacuum tests (thermal shock) were carried out on the rf feedthroughs with success.

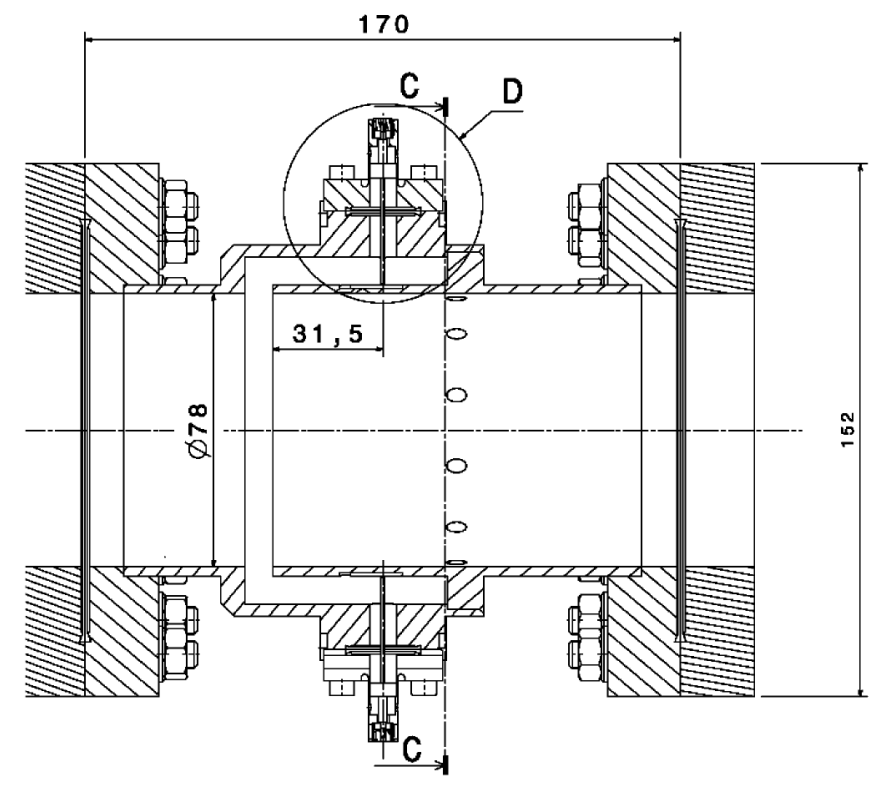

FIG. 5. Drawing of the modified cavity BPM.

One of the biggest problems on the cavity installed in the cryomodule was the cleaning. As the BPM is designed to be used in a clean environment, twelve holes of $5 \mathrm{~mm}$ diameter are drilled at the end of the reentrant part for more effective cleaning. Cleaning tests were successfully performed at DESY and validated the system for the cleaning.

The resonant cavity was, first, simulated with the software HFSS (Ansoft) to determine its frequencies, loaded quality factors, and $R / Q$ ratio for each resonant mode. Figure 6 shows electrical field, for the monopole and dipole modes, is high in the coaxial cylinder and the gap.

The rf measurements, presented in Table I, compare some computed quantities to measured values and give information on the sensitivity of the rf characteristics to the machining, mounting, and operating environments.

The loaded quality factors $Q_{l}$ are determined by HFSS with matched feedthroughs in eigensolver mode. In using MATLAB [12] and the HFSS calculator, the $R / Q$ ratio was computed with Eq. (3). The monopole and dipole modes are around 1.25 and $1.72 \mathrm{GHz}$ and the loaded quality factors are quite low. The unloaded quality factor for the monopole and dipole modes were calculated with HFSS and are, respectively, 510 and 625 . The external quality factor, defined in Eq. (6), is equal to the loaded quality factor. The antenna position reduces the magnetic loop coupling and increases the separation of the rf modes (monopole and dipole modes). Monopole and dipole signals have a better distinction and the rejection of the monopole signal is easier.

The reentrant cavity BPM was installed in a warm part in the FLASH linac at DESY, as illustrated Fig. 7, to be tested with beam.

Figure 8 shows the Fourier transform of a signal on the output of one pickup. The step around $3 \mathrm{GHz}$ fits with the 

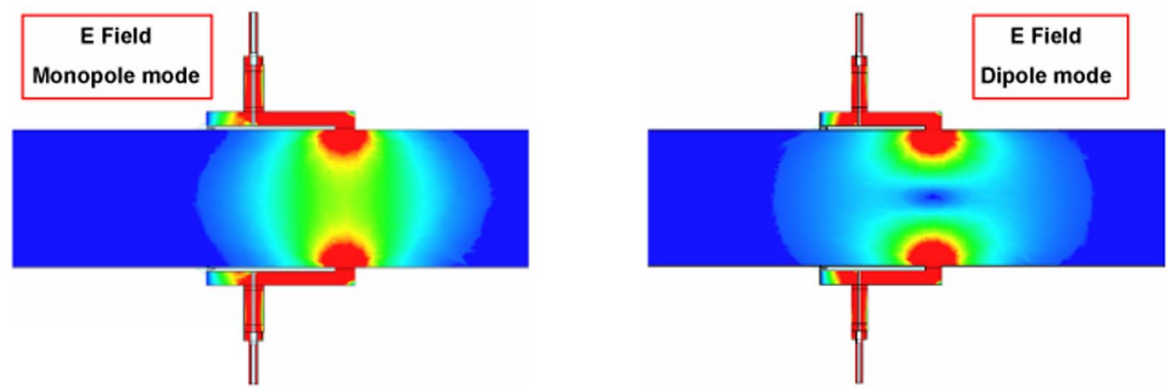

FIG. 6. (Color) Electrical fields for the monopole and dipole modes.

TABLE I. The rf characteristics of the new reentrant cavity BPM.

\begin{tabular}{lcccccc}
\hline \hline Eigenmodes & $F(\mathrm{MHz})$ & $F(\mathrm{MHz})$ & $Q_{l}$ & $Q_{l}$ & $R / Q(\Omega) 5 \mathrm{~mm}$ offset & $R / Q(\Omega) 10 \mathrm{~mm}$ offset \\
\hline & Calculated & Measured & Calculated & Measured & Calculated & Calculated \\
Monopole mode & 1250 & 1255 & 22.95 & 23.8 & 12.9 & 12.9 \\
Dipole mode & 1719 & 1724 & 50.96 & 59 & 0.27 & 1.15 \\
\hline \hline
\end{tabular}

$2.94 \mathrm{GHz}$ cutoff frequency of the beam pipe mode $\left(\mathrm{TM}_{01}\right)$. The modes, having a frequency above can propagate in the beam pipe. Conversely, the disturbances above the cutoff frequency from elsewhere can also propagate down to the BPM.

The transmission measurement on the opposite antennas, illustrated in Fig. 9, was completed in the 1 to $4 \mathrm{GHz}$ range. All peaks correspond to eigenmodes present in the reentrant cavity. The first and second peaks are the monopole and dipole modes, the others are higher order modes which can propagate out of the cavity through the beam pipe. The $1.72 \mathrm{GHz}$ band pass filter, used in the signal processing, was measured in laboratory and at $3 \mathrm{GHz}$ its attenuation is around $-70 \mathrm{~dB}$ and around $-60 \mathrm{~dB}$ at

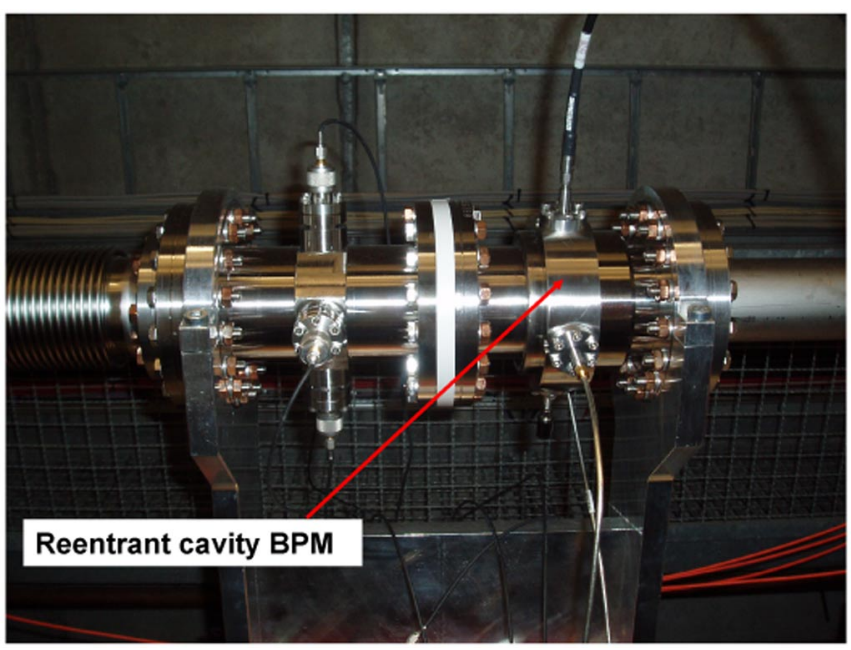

FIG. 7. (Color) Reentrant cavity BPM installed in the FLASH linac.
$4 \mathrm{GHz}$. These "higher order modes" should be therefore well rejected.

Figure 10 represents the signal measured on one pickup in the time base with a $5 \mathrm{GHz}$ bandwidth scope. It shows a clean separation between two macropulses distant to $1 \mu \mathrm{s}$. No long range echo is observed, confirming the possibility for measurements in a multibunches mode.

Because of the finite tolerances in machining, welding, and mounting, some small distortions of cavity symmetry are generated. A displacement of the beam in the $x$ direction gives not only a reading in that direction but also a nonzero reading in the orthogonal direction $y$. This asymmetry is called cross talk. Cross-talk isolation measure-

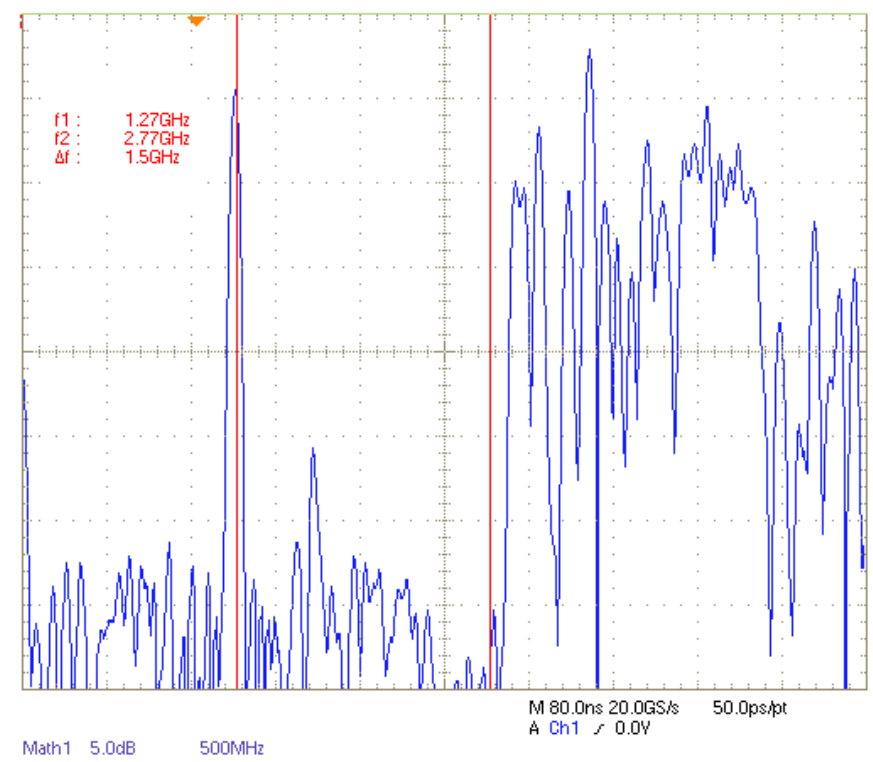

FIG. 8. (Color) Pickup signal spectrum with $0.8 \mathrm{nC}$ beam charge and a macropulse repetition rate around $5 \mathrm{~Hz}$. 


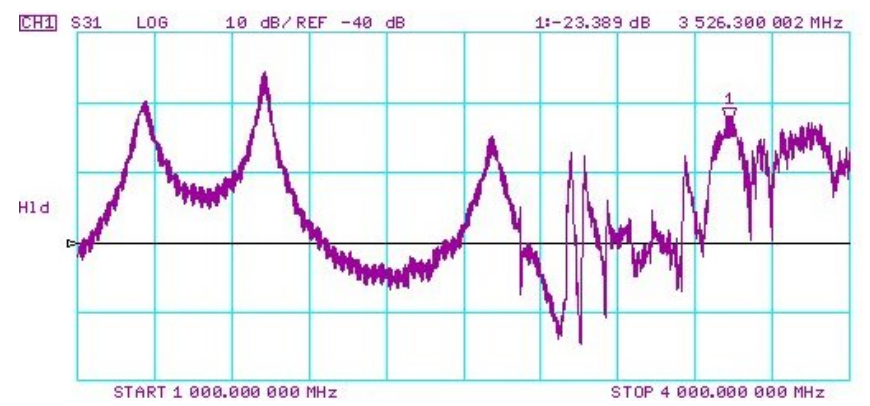

FIG. 9. (Color) Transmission measurement on the two opposite antennas without beam.

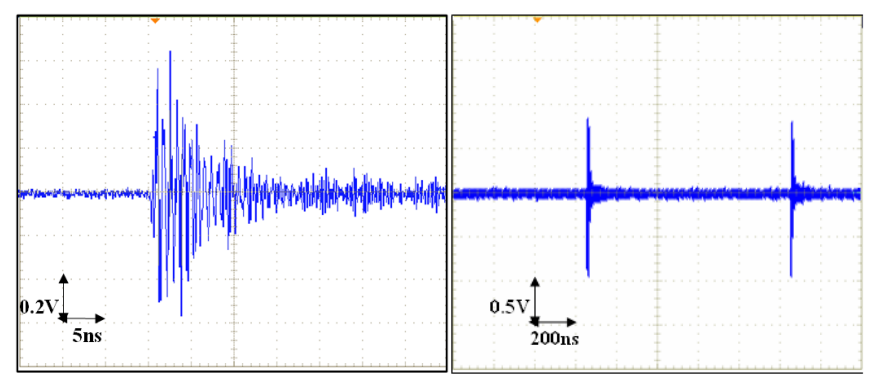

FIG. 10. (Color) Signal from one pickup.

ments are performed on the reentrant cavity with a network analyzer [11]. From the measurements, the cross-talk isolation value is estimated about $33 \pm 1 \mathrm{~dB}$ measured in the tunnel.

\section{B. The rf signal processing electronics}

The signal processing uses a single stage downconversion to obtain $\Delta / \Sigma$ and is shown in Fig. 11. It is composed of standard rf components: hybrid couplers, phase shifters, filters, isolators, and mixers.

The $\Delta$ and $\Sigma$ signals are obtained from a passive fourports $180^{\circ}$ hybrid. The $180^{\circ}$ hybrid coupler is connected to each pair of opposite antennas with some $75 \mathrm{~m}$ semirigid cables. The rejection of the monopole mode, on the $\Delta$ channel, proceeds in three steps: (i) a mode symmetry based rejection with a hybrid coupler. Its isolation is higher than $20 \mathrm{~dB}$ in the range of 1 to $2 \mathrm{GHz}$. A local enhancement of the isolation to be about $30 \mathrm{~dB}$ at $1.25 \mathrm{GHz}$ is obtained with additional external phase shifters; (ii) a frequency domain rejection with a band pass filter centered at the dipole mode frequency. Its bandwidth of $110 \mathrm{MHz}$ also provides a noise reduction; (iii) a synchronous detection of the dipole mode signal. A $9 \mathrm{MHz}$ reference signal, from the linac control system is combined with a phase locked loop to generate a local oscillator signal (LO) for the mixers at the dipole mode frequency. Phase tuning for the synchronous detection is refined while visualizing the delta/sigma signal on a scope and the gain of each channel was adjusted to avoid saturation from ADCs.

On the $\Sigma$ channel, a direct detection is used. This signal is used in order to normalize the $\Delta$ signal, which determines the position of the beam. This normalization is made by a control system. Signals detected by the signal processing electronics extract the beam position (displacement) and deliver this information to the acquisition board. The sampling and the interface to the control system are carried with 14 bit ADCs digital electronics [13].

\section{Time resolution}

One of the most important parameters for a BPM is the time resolution, which is usually identified to the damping time and is given by the following formula:

$$
\tau=\frac{1}{\pi B}
$$

where $B$ is the bandwidth in hertz, defined by the relation

$$
B=\frac{f_{d}}{Q_{l d}}
$$

with $f_{d}$ the frequency and $Q_{l d}$ the loaded quality factor for the dipole mode. The time resolution is therefore around $9.5 \mathrm{~ns}$ for the reentrant BPM.

If the system (cavity + signal processing) is studied, the length of the signal for the reentrant BPM behind the signal

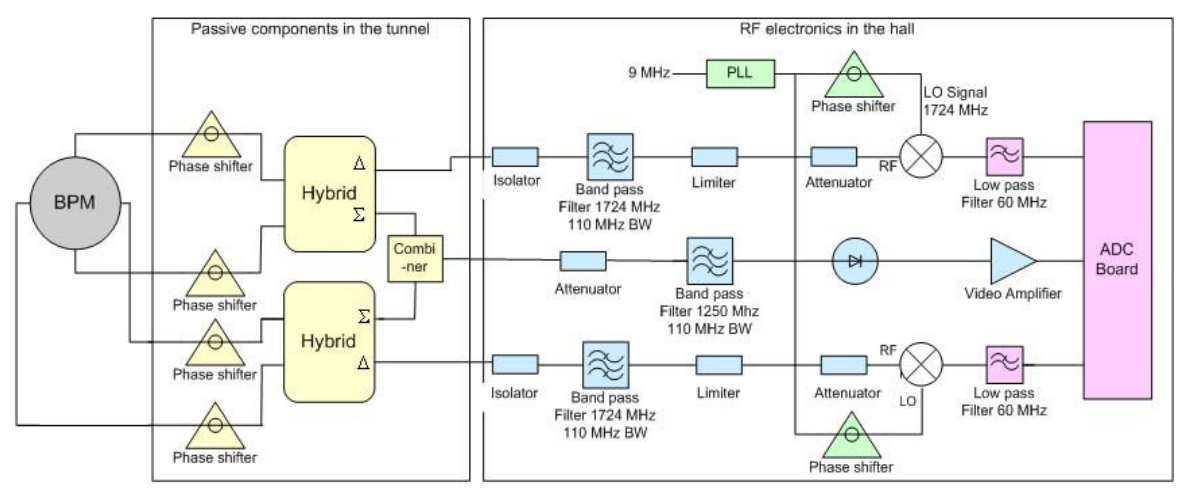

FIG. 11. (Color) The rf signal processing electronics. 


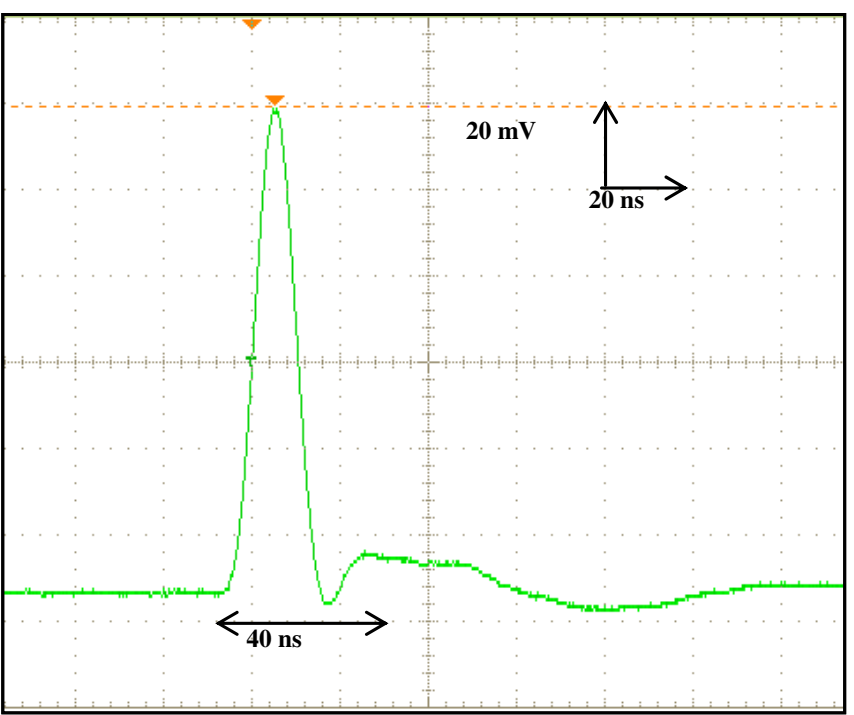

FIG. 12. (Color) Output signal from the signal processing.

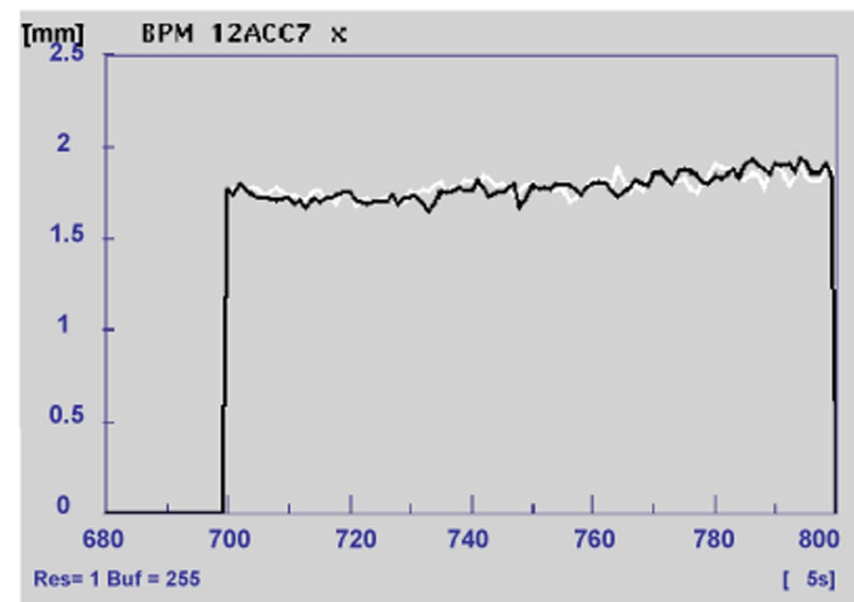

FIG. 13. (Color) Position of 100 bunches in a macropulse read by the reentrant BPM.

processing electronics is considered as the time resolution. It was measured around $40 \mathrm{~ns}$ with a scope as shown Fig. 12.

This time is lower than the separation between bunches on the FLASH linac (110 ns); the bunch to bunch measurements are therefore possible as illustrated in Fig. 13 where the position of each bunch is read by the reentrant BPM.

\section{Estimation of the performances for the reentrant BPM}

The position resolution is the minimum position difference that can be statistically resolved. It is an rms value related to the signal to noise ratio in the system. The signal voltage of the BPM is determined by the beam's energy loss to the resonant mode and by the external coupling of the coaxial cable.

To assess the performance of the system, a model (cavity + signal processing) is elaborated with a MATHCAD [14] code based on Fourier transforms. The simulation covers a span from 0 to $20 \mathrm{GHz}$. A resonant given mode in the cavity is modeled as a resonant circuit with an electrical circuit consisting of a resistor (R), an inductor (L), and a capacitor (C). The delivered time domain signal $\left(S_{i}\right)$ is determined by Eq. (7) and is calculated as

$$
S_{i}(t)=\Phi(t)\left[V_{i} \exp \left(-\frac{\omega_{i} t}{2 Q_{\mathrm{ext}}}\right) \cos \left(a_{i} t-\frac{\omega_{i} \sin \left(a_{i} t\right)}{2 Q_{\mathrm{ext} i} a_{i}}\right)\right]
$$

with

$$
a_{i}=\omega_{i} \sqrt{1-\frac{1}{4 Q_{\mathrm{ext} i}{ }^{2}}} \quad \text { and } \quad V_{i}=\sqrt{\frac{\omega_{i}^{2}(R / Q)_{i} q^{2} Z}{\zeta_{i} Q_{\mathrm{ext} i}}},
$$

where $\Phi(t)$ is the Heaviside function, $q$ is the bunch charge, $Z$ is the $50 \Omega$ cable impedance, $\omega_{i}$ is the frequency, $Q_{\text {ext } i}$ is external quality factor of each mode, $(R / Q)_{i}$ circuit defines the coupling to the beam and $\zeta_{i}=4$ if it is a monopole mode or $\zeta_{i}=2$ if it is a dipole mode.

The signal from a pickup ( $\left.S_{\text {pickup }}\right)$ is the sum of all resonant modes excited by the beam:

$$
S_{\text {pickup }}=\sum S_{i}
$$

To simulate the signal processing, the transfer functions of different components are used. Figure 14 gives a description of the method for the $\Delta$ channel.

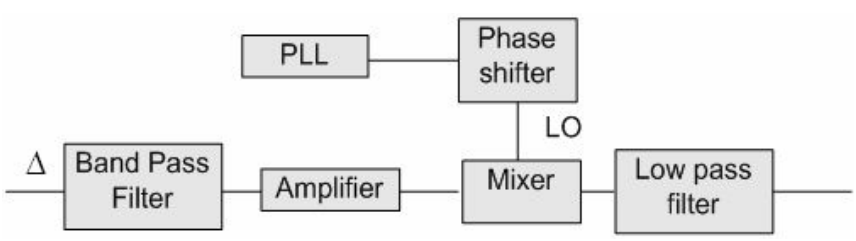

FIG. 14. $\Delta$ channel signal processing.

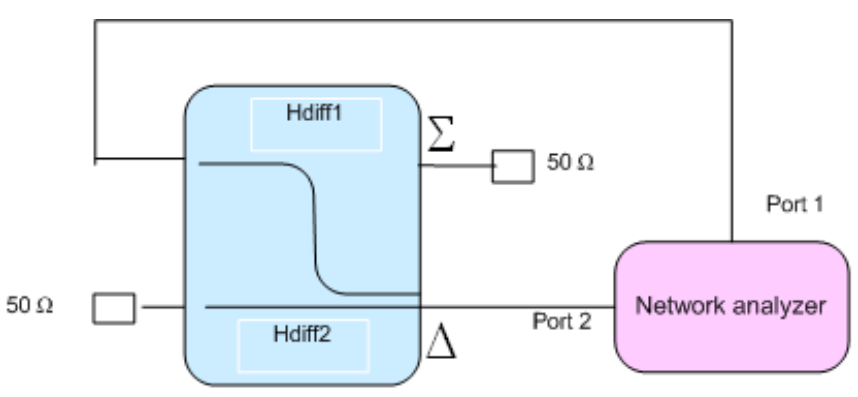

FIG. 15. (Color) $S$ parameters measurement of the hybrid $180^{\circ}$. 


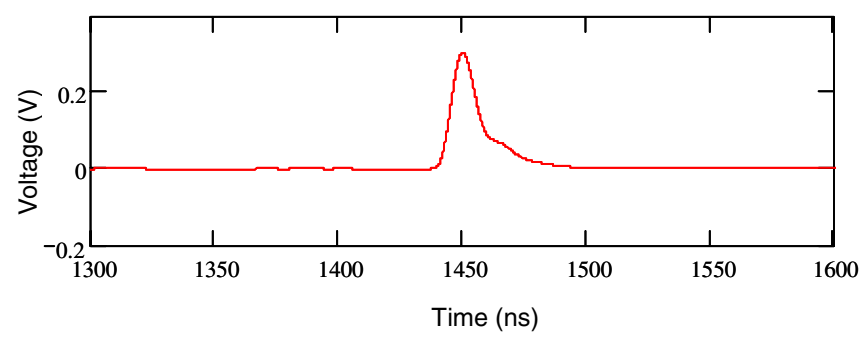

FIG. 16. (Color) IF simulated signal from the $\Delta$ channel with $10 \mathrm{~mm}$ beam offset.

The transfer function of cables $\left(H_{c}\right)$ takes into account the effect of attenuation, dispersion, and the length of cables (here $75 \mathrm{~m}$ ). The model of the $180^{\circ}$ hybrid couplers composing the signal processing is derived from measurements with a network analyzer: the transmission measurement from the port 1 to port $\Delta$, as shown Fig. 15, gives the transfer function " $H_{\text {diff } 1}$," the transmission from the port 2 to the port $\Delta$ gives " $H_{\text {diff2." }}$ " The same measurements are

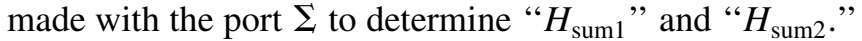

The hybrid isolation is determined by the following relation:

$$
I_{\text {hybrid }}=20 \log \left(\left|H_{\text {diff } 1}+H_{\text {diff } 2}\right|\right) .
$$

Those hybrid couplers have an isolation higher than $20 \mathrm{~dB}$ in the passband of $1-2 \mathrm{GHz}$ but are different in details especially outside the passband. The $\Delta$ signal from the hybrid is given by the following relation:

$$
T_{\Delta}=\left[\left(S_{m}-S_{d}\right) H_{c} H_{\text {diff } 1}\right]+\left[\left(S_{m}+S_{d}\right) H_{c} H_{\text {diff } 2}\right] \text {. }
$$

To validate the model, the "sum" signal peak power was measured around $36 \mathrm{dBm}$ for $0.9 \mathrm{nC}$, it is of the same order of magnitude compared to simulations.

The band pass filter has a $110 \mathrm{MHz}$ bandwidth centered at $1.72 \mathrm{GHz}$. Its transfer function is given by a CAD code [15]. The local oscillator (LO) signal is modeled by a sine wave at the dipole frequency with $1 \mathrm{~V}$ amplitude. A phase shift is added to adjust in phase the LO signal and the rf signal (without monopole mode) from the $\Delta$ channel. Then a $60 \mathrm{MHz}$ lowpass filter, which the transfer function is given by the same CAD code, is used to determine the IF signal. This signal (with monopole mode), illustrated in Fig. 16, is then sampled at the peak for a significant beam offset.

The position resolution is limited by the signal to noise ratio of the system. The noise comes from the thermal noise and some components used in the signal processing electronics. The thermal noise of a system is given by the following equation:

$$
P_{\mathrm{th}}=k_{b} T B,
$$

where $k_{b}$ is Boltzmann's constant, $B$ is defined by the bandwidth of the signal processing channel in hertz, and $T$ is the room temperature in kelvin.

The noise level present at the output of the cavity BPMs is amplified by the devices which compose the signal processing electronics. To calculate the noise level, the thermal noise is added to the noise factor and to the gain. The noise level is therefore given by the following equation:

$$
P_{n}=\mathrm{NFG} P_{\mathrm{th}},
$$

where NF is the total noise figure of the signal processing channel, $G$ is the gain of the signal processing, and $P_{\text {th }}$ is the thermal noise. The total noise introduced into the system by the electronics can be evaluated by the noise figure in a cascaded system and is applied by the following formula:

$$
\mathrm{NF}=F_{1}+\frac{F_{2}-1}{G_{1}}+\frac{F_{3}-1}{G_{1} G_{2}}+\cdots,
$$

where NF is the total noise factor of the signal processing, $F_{i}$ and $G_{i}$ are, respectively, the noise factor and the gain of component $i$.

The thermal noise power is defined by the lowpass filter bandwidth, i.e., bandwidth is equal to $60 \mathrm{MHz}$ in Eq. (14). The noise amplitude in the hall was measured on ADC board with a scope to be about $200 \mu \mathrm{V}$ peak. The rms environment noise is therefore around $65 \times 10^{-6} \mathrm{~V}$ that limits the resolution for the reentrant BPM. As the limitation of the resolution is due to the ADCs noise, to improve the resolution, the dynamic range has to be reduced. That is why, if an amplifier with a gain around $18 \mathrm{~dB}$ and a noise figure around $3.8 \mathrm{~dB}$ is added in the $\mathrm{rf}$ signal processing channel, this system gets a resolution better than $1 \mu \mathrm{m}$ with a dynamic range limited to $\pm 1 \mathrm{~mm}$.

Table II gives the signal amplitude behind the low pass filter with $10 \mathrm{~mm}$ and $1 \mathrm{~mm}$ beam offset, the total noise and the resolution.

\section{E. Beam measurements}

The first beam tests of the reentrant BPM, installed in the warm part in the FLASH linac, were operated in single bunch with a charge around $1 \mathrm{nC}$. The charge of the reentrant BPM was calibrated thanks to the toroids. An offset on $\Delta x$ and $\Delta y$ channels was added in the acquisition

TABLE II. Signal and noise level calculated for the reentrant BPM.

\begin{tabular}{lccc}
\hline \hline Systems & Signal on $\Delta$ channel $(\mathrm{mV})$ & Total noise $(\mathrm{mV})$ & Simulated resolution $(\mu \mathrm{m})$ \\
\hline BPM with $10 \mathrm{~mm}$ beam offset & 181 & $65 \times 10^{-3}$ & 3.65 \\
BPM with $1 \mathrm{~mm}$ beam offset & 149 & $65 \times 10^{-3}$ & 0.45 \\
\hline \hline
\end{tabular}



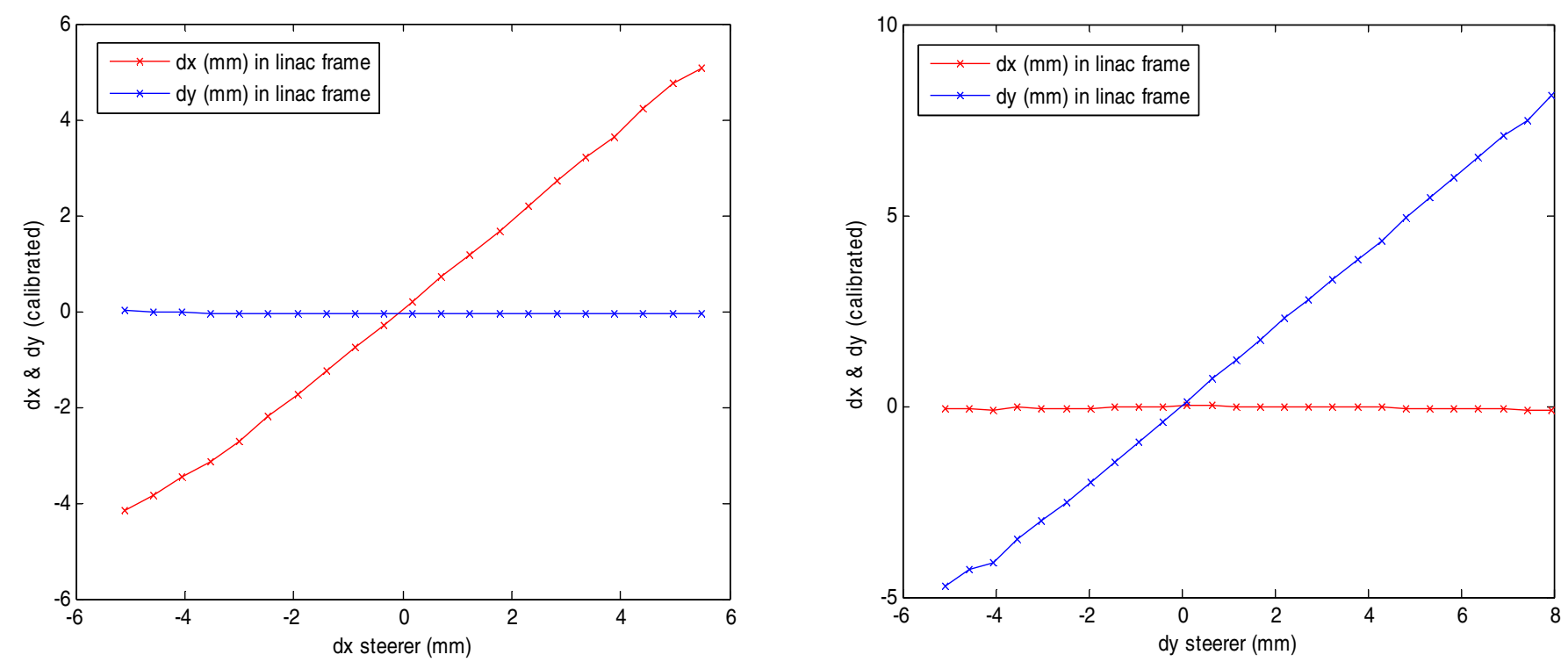

FIG. 17. (Color) Calibration results from horizontal (left) and vertical (right) steerings.

software to read a zero in this condition. Another calibration coefficient was computed from a linear fit of the predicted position to the measured position. The relative beam displacement $(\Delta x)$ at the BPM location was calculated in using a transfer matrix $\left(R_{12}\right)$ between steerers and BPM (made of drifts) for different values of drive current in the steerers: $\Delta x=R_{12} \Delta x^{\prime}$ (where $\Delta x^{\prime}$ is the beam angle at steerer). The steerers were used to move the beam, and the magnets between the steerers and the BPM were switched off to reduce errors and simplify calculation. An average of 500 points for each steerer setting was used.

First, as illustrated in Fig. 17, the deviation range was limited to $\pm 5 \mathrm{~mm}$ for a more accurate calibration.

To cancel the beam jitter, the BPM resolution has been calculated from cross correlations with the other BPMs installed in the FLASH linac. The reentrant BPM resolution is, therefore, measured by correlating the reading of the reentrant BPM in one plane against the readings of all other BPMs in the same plane [16]. The resolution, illustrated Fig. 18, is, on the vertical channel ( $Y$ channel), around $4 \mu \mathrm{m}$ and on the horizontal channel ( $X$ channel) around $8 \mu \mathrm{m}$, limited only by the electromagnetic contamination in the experimental hall. Those results are quite similar to the theoretical resolution calculated around $3.65 \mu \mathrm{m}$ with $10 \mathrm{~mm}$ beam offset.

To improve the linearity of dynamic range, a $6 \mathrm{~dB}$ attenuator was added on $X$ and $Y$ channels. As shown in Fig. 19, dynamic range is linear on $\pm 12 \mathrm{~mm}$.

Table III shows a comparison of the resolution with different charge values and between a system with and without $6 \mathrm{~dB}$ attenuator.

With a $6 \mathrm{~dB}$ attenuator, the dynamic range is improved but the resolution is damaged. As shown with Eq. (7), with a low charge the signal is lower and therefore the resolution is damaged. In the future, to keep the same resolution, some variable attenuators could be used.

\section{CONCLUSION}

This paper describes the performance of a reentrant cavity beam position monitor which is designed to be used inside a cryomodule, in a clean environment where dust particle contamination has to be avoided. Its main features are the small size of the rf cavity, a large aperture $(78 \mathrm{~mm})$, an excellent linearity, a time resolution around $40 \mathrm{~ns}$, and the beam charge measurement. A first prototype

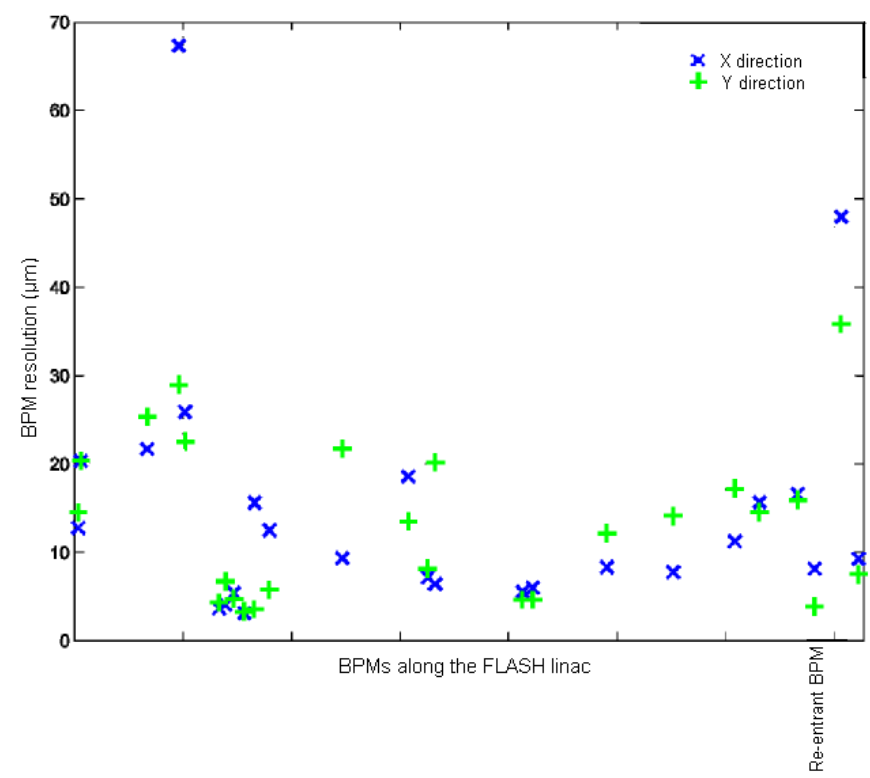

FIG. 18. (Color) Resolution calculated for the reentrant BPM installed in the warm part of the FLASH linac. 

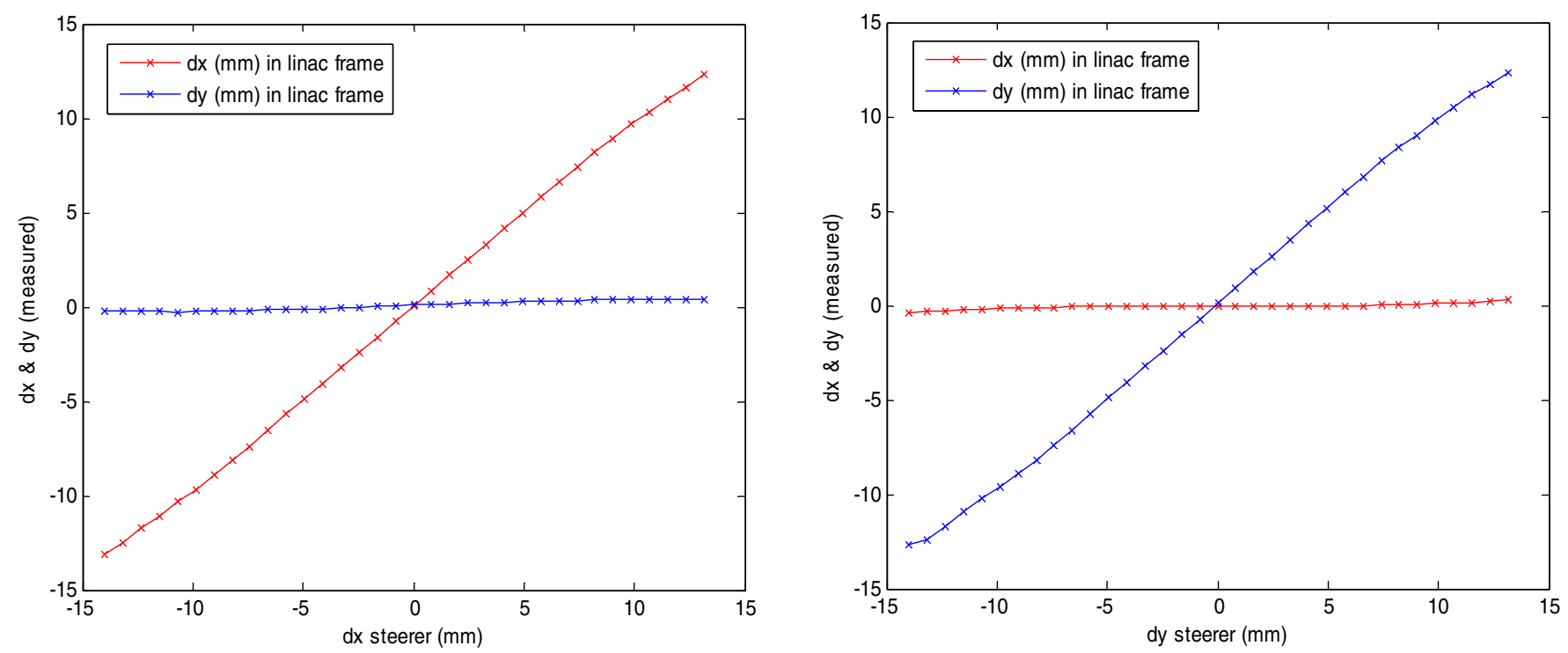

FIG. 19. (Color) Position read by the reentrant BPM vs the predicted position from horizontal (left) and vertical (right) steerings with $1 \mathrm{nC}$ charge.

TABLE III. Resolution of the reentrant BPM.

\begin{tabular}{lcc}
\hline \hline Charge $(\mathrm{nC})$ & $\mathrm{BPM}(\mu \mathrm{m})$ & $\mathrm{BPM}+6 \mathrm{~dB}$ attenuator $(\mu \mathrm{m})$ \\
\hline 1 & $\sim 4$ & $\sim 7$ \\
0.8 & & $\sim 12$ \\
0.5 & $\sim 11.8$ & $\sim 21$ \\
0.2 & $\sim 30.1$ & $\sim 55$ \\
\hline \hline
\end{tabular}

has already proved its operation at cryogenic temperature inside a cryomodule. The second installed on the warm section in the FLASH linac showed a high linearity in the range of $\pm 12 \mathrm{~mm}$, a high position resolution better than $10 \mu \mathrm{m}(4 \mu \mathrm{m}$ for the $Y$ channel and $8 \mu \mathrm{m}$ for the $X$ channel), and the possibility to carry out some bunch to bunch measurements.

Future work towards a few reentrant cavity BPMs which will be installed in XFEL cryomodules will include modification of the design to improve alignment between the BPM and quadrupole. Some variable attenuators will be added in the signal processing electronics to have a dynamic range $\pm 15 \mathrm{~mm}$ and operate with a resolution better than $10 \mu \mathrm{m}$.

\section{ACKNOWLEDGMENTS}

We acknowledge the support of the European Community-Research Infrastructure Activity under the FP6 "Structuring the European Research Area" program (CARE, Contract No. RII3-CT-2003-506395). We thank our colleagues from DESY: the TTF operational team for its support, MDI and MVP teams for their help on the DESY site and fruitful discussions on the BPMs. We would like, too, to thank all our colleagues from CEA Saclay for their support on this project.

[1] V. Ayvazyan et al., Eur. Phys. J. D 37, 297 (2006).

[2] J. Rossbach, in Proceedings of the 10th European Particle Accelerator Conference, Edinburgh, Scotland, 2006 (EPS$A G$, Edinburgh, Scotland, 2006).

[3] DESY Reports: No. DESY-02-167 and No. DESYTESLA-FEL-2002-09, edited by R. Brinkmann et al., 2002.

[4] R. D. Heuer, Nucl. Phys. B, Proc. Suppl. 154, 131 (2006).

[5] DESY Reports: No. DESY-01-011 and No. DESYTESLA-2001-23, edited by R. Brinkmann et al., 2001.

[6] R. Bossart, in Proceedings of the 17th International Linear Accelerator Conference (LINAC94), Tsukuba, Japan, 1994 (KEK, Japan, 1994).

[7] P. Wilson, SLAC-PUB-2884, 1991.

[8] C. Magne et al., in Proceedings of the 19th International Linear Accelerator Conference (LINAC98), Chicago, IL, 1998.

[9] C. Magne et al., in Proceedings of the 9th Beam Instrumentation Workshop (BIW2000), Cambridge, MA, 2000, AIP Conf. Proc. No. 546 (AIP, Cambridge, MA, 2000).

[10] HFSS, Ansoft Corporation http://www.ansoft.com/.

[11] C. Simon et al., in Proceedings of the 12th Beam Instrumentation Workshop (BIW2006), Batavia, IL, 2006, AIP Conf. Proc. No. 868 (AIP, Batavia, IL, 2006).

[12] MATLAB, The Mathworks, http://www.mathworks.com/.

[13] J. Andruszkow, P. Jurkiewicz, and F. Tonisch, 8-channel FastADC with 14 bit resolution, Reference Manual.

[14] MATHCAD 13, http://www.ptc.com/products/mathcad/.

[15] Filter Free, Nuhertz Technologies, http://www.nuhertz. $\mathrm{com} /$.

[16] N. Baboi et al., in Ref. [11]. 